\title{
Fc receptor-mediated phagocytosis in tissues as a potent mechanism for preventive and therapeutic HIV vaccine strategies
}

\author{
M Sips $^{1,2}$, M Krykbaeva ${ }^{1}$, TJ Diefenbach ${ }^{1}$, M Ghebremichael $^{1}$, BA Bowman ${ }^{1}$, A-S Dugast ${ }^{1}$, AW Boesch $^{3}$, \\ H Streeck ${ }^{4}$, DS Kwon ${ }^{1}$, ME Ackerman ${ }^{3}$, TJ Suscovich ${ }^{1}$, P Brouckaert $^{2}$, TW Schacker ${ }^{5}$ and G Alter ${ }^{1}$
}

Although the development of a fully protective HIV vaccine is the ultimate goal of HIV research, to date only one HIV vaccine trial, the RV144, has successfully induced a weakly protective response. The $31 \%$ protection from infection achieved in the RV144 trial was linked to the induction of nonneutralizing antibodies, able to mediate antibodydependent cell-mediated cytotoxicity (ADCC), suggestive of an important role of Fc-mediated functions in protection. Similarly, Fc-mediated antiviral activity was recently shown to play a critical role in actively suppressing the viral reservoir, but the Fc effector mechanisms within tissues that provide protection from or after infection are largely unknown. Here we aimed to define the landscape of effector cells and Fc receptors present within vulnerable tissues. We found negligible Fc receptor-expressing natural killer cells in the female reproductive and gastrointestinal mucosa. Conversely, Fc receptor-expressing macrophages were highly enriched in most tissues, but neutrophils mediated superior antibodymediated phagocytosis. Modifications in Fc domain of VRC01 antibody increased phagocytic responses in both phagocytes. These data suggest that non-ADCC-mediated mechanisms, such as phagocytosis and neutrophil activation, are more likely to play a role in preventative vaccine or reservoir-eliminating therapeutic approaches.

\section{INTRODUCTION}

Although a vaccine that is able to induce broadly neutralizing antibodies (bnAbs) against HIV remains a top priority, to date no vaccine strategy has induced antibodies with appreciable neutralizing coverage of global viral quasispecies. However, a reduced risk of infection and/or enhanced viral control after infection has been observed in both human ${ }^{1}$ and non-human primate (NHP) studies, ${ }^{2,3}$ both associated with the induction of nonneutralizing antibodies with potent Fc-mediated effector functions. In addition, although the broadly neutralizing antibody b12 was able to provide sterilizing protection from infection, the protective efficacy of this monoclonal was partially lost upon the ablation of FcR-binding activities. ${ }^{4}$

Beyond their role in preventing infection, Fc effector functions have also been implicated in postinfection control and clearance of viral infections including Ebola ${ }^{5}$ and Influenza. ${ }^{6}$ Similarly, antibody effector function has been linked to spontaneous and durable control of viral load in HIV infection. $^{7,8}$ Importantly, control of the viral reservoir is critically dependent on the Fc effector functions of bnAbs, as loss of Fc effector activity leads to the rapid loss of viral control despite potent neutralizing activity. ${ }^{9}$ Thus, although delivery of potent neutralizing antibodies drives a transient reduction in plasma viremia in both $\mathrm{NHP}^{10}$ and humans, ${ }^{11}$ the viral reservoir nearly always rebounds because of incomplete antibody-mediated eradication of reactivated cells, suggesting that the enhanced Fc-mediated antibody effector functions, able to recruit innate immune killing, particularly at sites where the reservoir hides, may enhance the elimination of infected cells.

Antibodies are able to recruit a wide array of antiviral functions via interactions between the crystallizable Fc fragment and Fc receptors, complement, or lectin-like receptors found on innate immune cells. ${ }^{12}$ Fc receptors exist for each

\footnotetext{
${ }^{1}$ Ragon Institute of MGH, MIT, and Harvard, Cambridge, Massachusetts, USA. ${ }^{2}$ Department of Biomedical Molecular Biology, Ghent University, Ghent, Belgium. ${ }^{3}$ Thayer School of Engineering at Dartmouth College, Hanover, New Hampshire, USA. ${ }^{4}$ Institute of HIV Research, University Hospital Essen, University of Duisburg-Essen, Essen, Germany and ${ }^{5}$ Division of Infectious Diseases, University of Minnesota, Minneapolis, Minnesota, USA. Correspondence: G Alter (galter@mgh.harvard.edu) 
of the immunoglobulin isotypes and are involved in directing disparate innate immunological functions that range from direct antiviral activity via phagocytosis, cellular cytotoxicity, complement activation, cytokine secretion to indirect immune regulation via the direction of anti-inflammatory responses often aimed at dampening inflammation. ${ }^{13}$ Thus, Fc receptors link the specificity of the adaptive immune system to the powerful effector functions of the innate immune system. However, because both the distribution of innate immune cells ${ }^{14}$ and the range of $\mathrm{Fc}$-mediated effector functions they can mediate ${ }^{15}$ differs from tissue compartment to compartment, it is likely that the potency of any given monoclonal antibody will depend on the distribution of FcRs on distinct subsets of innate immune cells within target tissues.

Because HIV infections largely occur through mucosal membranes, ${ }^{16}$ and persistent reservoirs likely hide in lymphoid tissues ${ }^{17}$ and the intestinal tract, ${ }^{18}$ in this study we aimed to define the antibody effector functions available within mucosal and lymphoid tissues that can be harnessed in the context of future prophylactic/therapeutic strategies. Surprisingly, tissueresident natural killer (NK) cells expressed negligible levels of Fc receptors, suggesting that tissue-resident NK cells are unlikely to contribute to antibody-mediated protective immunity at the site of infection. However, Fc $\gamma$ RII- and Fc $\gamma$ RIIIexpressing macrophages and neutrophils were present in tissues collected from both HIV-seronegative and -seropositive subjects. Although tissue-resident neutrophils were less abundant, they mediated more effective phagocytic clearance of immune complexes. Neither macrophage- nor neutrophilmediated clearance was affected by inflammatory cytokines associated with enhanced risk of HIV acquisition. ${ }^{19}$ Moreover, Fc-engineered VRC01 antibodies drove improved mucosal phagocytic activity, offering a means to further increase innate immune antiviral activity aimed at preventing or clearing infection. These data strongly argue that antibody-driven functional activities mediated by cells other than NK cells are more likely to afford protection from infection as well as have therapeutic activity within mucosal and lymphoid tissues.

\section{RESULTS}

Differential NK cell, macrophage, and neutrophil distribution in mucosal tissues

Given that the distribution of innate immune cells may vary among different tissues, ${ }^{14,20}$ the biological activity of functional antibodies is highly dependent on the availability of each effector cell type at the site of transmission or viral replication. Thus, we first aimed to define the frequency and localization of these cells within healthy tissues, as this would represent the landscape of effector cells available to antibodies induced by a prophylactic vaccine or passive monoclonal therapeutic prevention strategy. Specifically, healthy samples from rectum, colon, vagina, cervix, uterus, and lymph node were stained for NKp46 or CD56 (NK cell markers), CD68 (macrophage marker), and 1-, 2-, and 3-defensins (resting neutrophil marker, with its expression limited to inactivated mature neutrophils) to determine the location of these innate effector cells.
Overall, the distribution of tissue-resident NK cells varied widely depending on the tissue type (Figure 1a). Rectum-, colon-, and vaginal mucosal-resident NK cells were found primarily in intraepithelial spaces, whereas NK cells in the endocervix and uterus were largely found in the mucosal lamina propria. Lymph node-resident NK cells were found sparsely distributed in the lymph node cortex, outside of follicles. Overall, the frequency of tissue-resident NK cells was variable (median cells per $\mathrm{mm}^{2}:$ colon $=29$, vagina $=27$, ectocervix $=8$, endocervix $=15$, uterine endometrium $=74$, lymph node $=9$ ), and relatively low in intestine and lymph node, with the greatest number in the uterine endometrium, where NK cells are known to be enriched and play an important role in fertilization and pregnancy. ${ }^{21}$

In contrast, macrophages were more prevalent in the gastrointestinal tract lamina propria, densely localized directly beneath the epithelium, and in the lymph node (median cells per $\mathrm{mm}^{2}:$ colon $=560$, lymph node $=500$, Figure $1 \mathbf{b}$ ). Other tissue compartments, including the upper genital tract, also showed slightly greater numbers of macrophages (median cells per $\mathrm{mm}^{2}$ : uterus endometrium $=153$, Figure 1d), with the exception of the lower female reproductive tract (FRT), where macrophages were less frequently observed (median cells per $\mathrm{mm}^{2}$ : vagina $=7$, ectocervix $=12$, endocervix $=17$ ).

Finally, we examined the frequency and location of neutrophils within the same tissues (Figure 1c). In general, neutrophils were the least abundant cell type in the rectal and colonic mucosa and in the upper female genital tract (median cells per $\mathrm{mm}^{2}$ : colon $=7$, endocervix $=10$, uterus $=3$ ); however, in vaginal tissue, often seen in epithelium, neutrophils were present at similar levels to NK cells and slightly outnumbered macrophages (median cells per $\mathrm{mm}^{2}=$ 39, Figure 1d). Lymph node neutrophils were found to be more abundant than NK cells, but less frequent than macrophages $($ median $=110)$.

Taken together, these data suggest distinct tissue-specific distribution profiles for each innate immune cell subset. Specifically, the data argue that macrophages are the most abundant innate immune cell-type within intestine and lymph node, whereas comparable levels of NK cells and neutrophils are observed in vaginal tissue, suggestive that distinct antibody-dependent mechanisms may play a dominant role at distinct portals of HIV entry as well as in curative strategies.

\section{Different FcR expression profiles in tissues}

$\mathrm{Fc}$ receptor expression varies depending on the maturation state of the cell as well as on its tissue localization. ${ }^{14}$ Thus, to gain deeper insights into the Fc receptor distribution on innate immune cells within tissue compartments, fresh colon and cervix specimens were collected from HIV-seronegative subjects. The tissues were mechanically disrupted and enzymatically digested to generate a cell suspension for flow cytometric interrogation using a panel of antibodies to detect Fc $\gamma$ RI (CD64), Fc $\gamma$ RII (CD32), Fc $\gamma$ RIII (CD16), and Fc $\alpha$ R (CD89) on NK cells, macrophages, and neutrophils. 
NK cells were defined as $\mathrm{FSC}^{\text {low }}, \mathrm{SSC}^{\text {low }}$, and CD45+ CD3 - CD56 + lymphocytes (Figure 2a,b). In contrast to blood NK cells, very few tissue-resident NK cells expressed Fc $\gamma$ RIII with slightly more Fc $\gamma$ RIII + NK cells observed in cervical tissues compared with colon. As expected, no Fc $\gamma \mathrm{RI}+$ or Fc $\alpha \mathrm{R}+$ colonic or cervical NK cells were detected; yet, variable but always detectable levels of Fc $\gamma$ RII + NK cells were observed in the gastrointestinal tract. Expression of functional CD32 molecules on human NK cells has been shown to be linked to an allelic polymorphism of the FCGRIIC gene that can drive the expression of functional FcR $\gamma$ IIc isoforms. ${ }^{22}$ This polymorphism may account for the high levels of FcR $\gamma \mathrm{II}+\mathrm{NK}$ cells observed in the two donor samples.

Mucosal macrophages were larger and more granular and were defined as FSC ${ }^{\text {intermed }}$, SSC ${ }^{\text {intermed }}$, and CD45 + CD3 $\mathrm{CD} 68+$ (Figure 2a,b). Two distinct populations of macrophages were consistently observed in colonic samples: $\mathrm{CD} 68^{\text {high }}$ and $\mathrm{CD} 68^{\text {low }}$ (Figure 2a). The majority of dominant CD68 ${ }^{\text {low }}$ macrophages expressed a single Fc $\gamma$ RII or no Fc $\gamma$ R, whereas $\sim 40 \%$ of the $\mathrm{CD} 68^{\text {high }}$ macrophages coexpressed Fc $\gamma \mathrm{RI} /$
Fc $\gamma$ RII or expressed Fc $\gamma$ RII alone (Figure 2f). Fc $\gamma$ RIII and $\mathrm{FcR} \alpha$ expression on total colonic macrophages was negligible (Figure 2c,e), with Fc $\gamma$ RIII restricted to $\mathrm{CD} 68^{\text {low }}$ and $\mathrm{Fc} \alpha \mathrm{R}$ restricted to $\mathrm{CD} 68^{\text {high }}$ macrophages (Figure 2c). In contrast, macrophages in the FRT exhibited a very different Fc receptor profile. Only CD $68^{\text {high }}$ macrophages were observed in cervical tissues (Figure 2b). Moreover, these macrophages expressed all $\mathrm{Fc}$ receptors frequently with a dominant expression of $\mathrm{FcR} \gamma \mathrm{II}$, followed by FcR $\gamma$ I detected on the majority (70\%) of cervical macrophages. FcR $\gamma$ III- and FcR $\alpha$-expressing macrophages were also more frequent in the FRT compared with gastrointestinal macrophages (Figure 2d,e). Notably, higher frequencies of Fc $\gamma \mathrm{R}$-coexpressing macrophages were found in the cervix compared with the gut, including dominant doublepositive Fc $\gamma \mathrm{RI}+\mathrm{Fc} \gamma \mathrm{RII}+$ macrophages and up to $20 \%$ of cervical macrophages expressing all three $\mathrm{Fc}$ receptors $(\mathrm{Fc} \gamma \mathrm{RI}+\mathrm{Fc} \gamma \mathrm{RII}+\mathrm{Fc} \gamma \mathrm{RIII}+)$, a population that was never observed in the intestinal tract. In summary, these data highlight the remarkable diversity in FcR expression on macrophages at distinct mucosal sites, as has been previously reported for lymphoid tissues. ${ }^{15}$
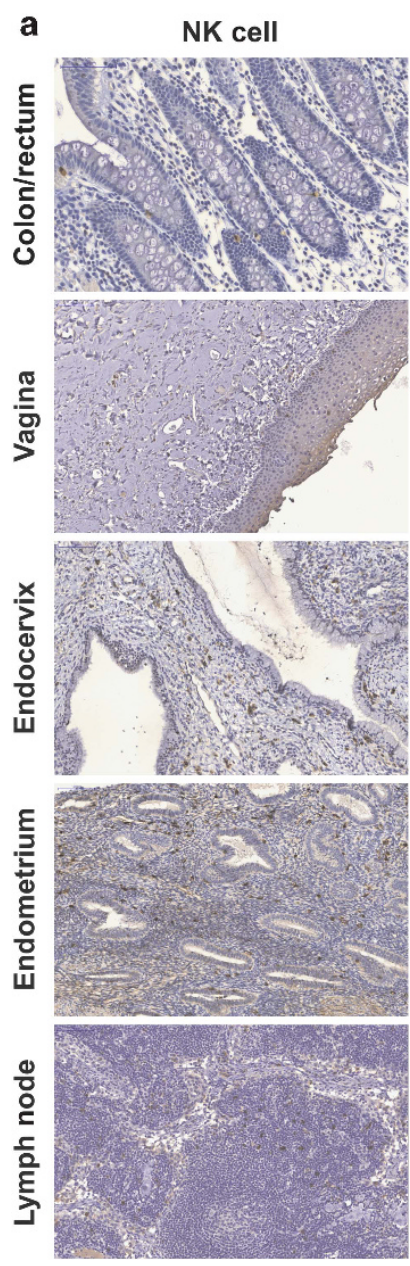

b
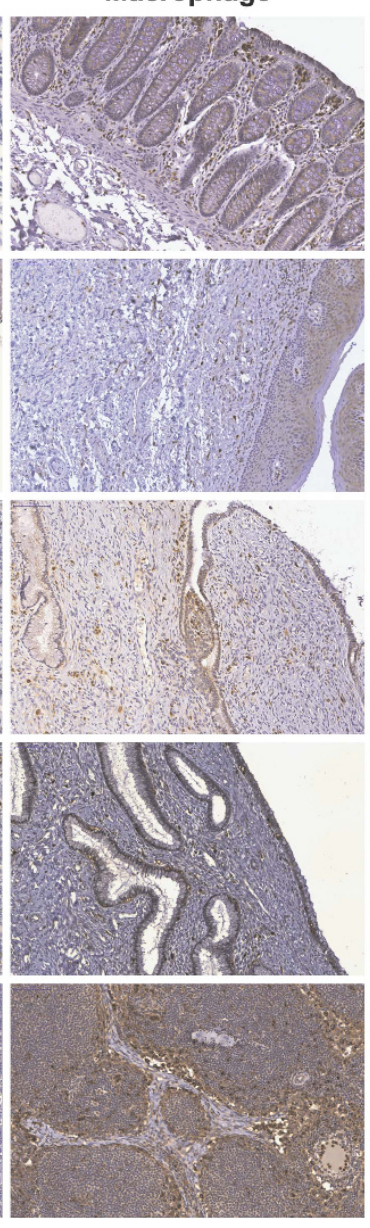

Figure 1 Localization of innate immune cells in tissues. (a) Natural killer (NK) cells, (b) macrophages (Ma), and (c) neutrophils (N) in colorectal mucosa, vagina, endocervix, uterus endometrium, and lymph node. Original magnification $\times 200$. Quantification of the cells is depicted in (d). NK cells, circles; macrophages, squares; neutrophils, triangles. Open shape, rectum; closed shape, colon. 
a

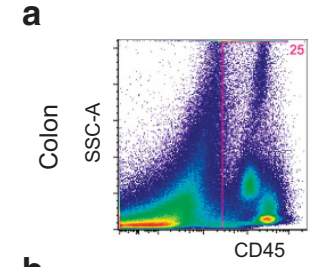

b

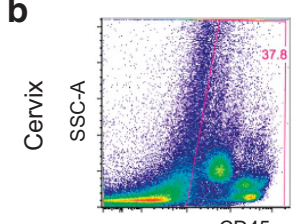

c

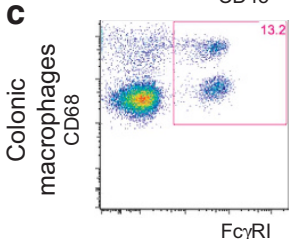

d

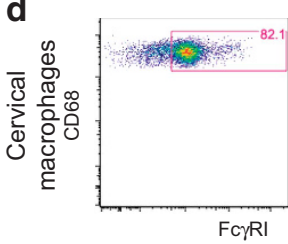

e

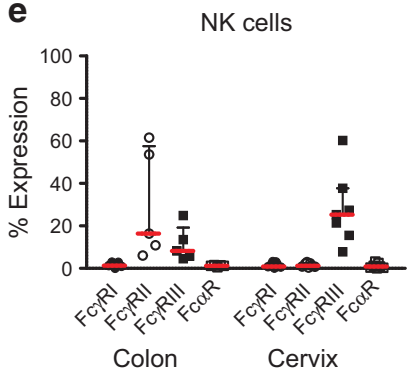

f

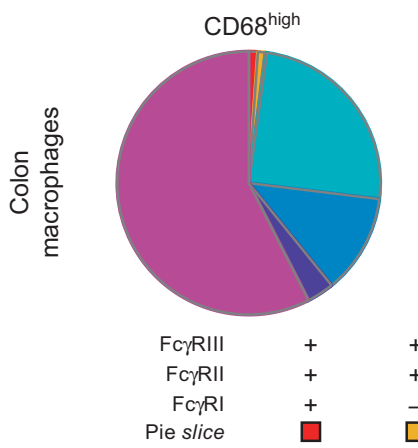

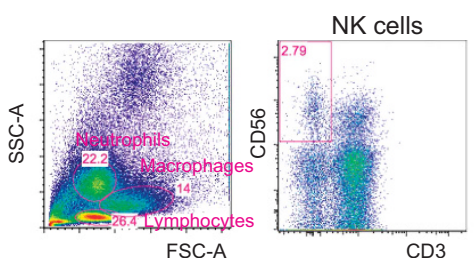
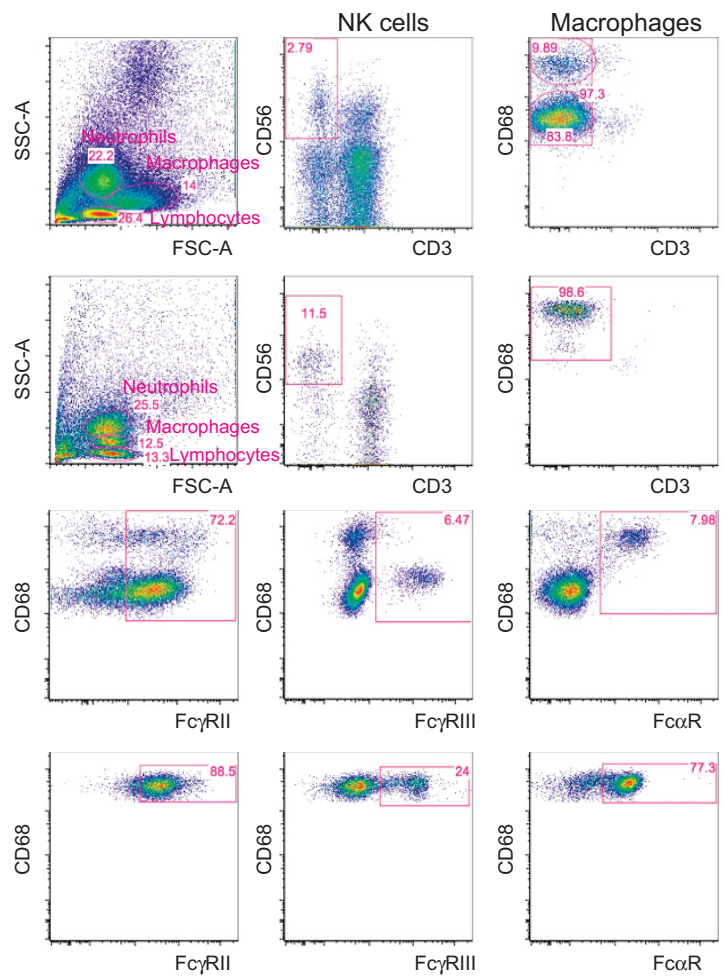

O

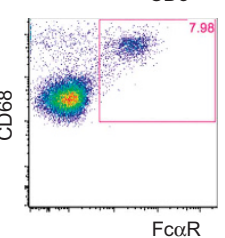

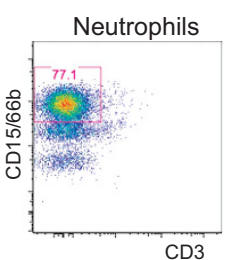
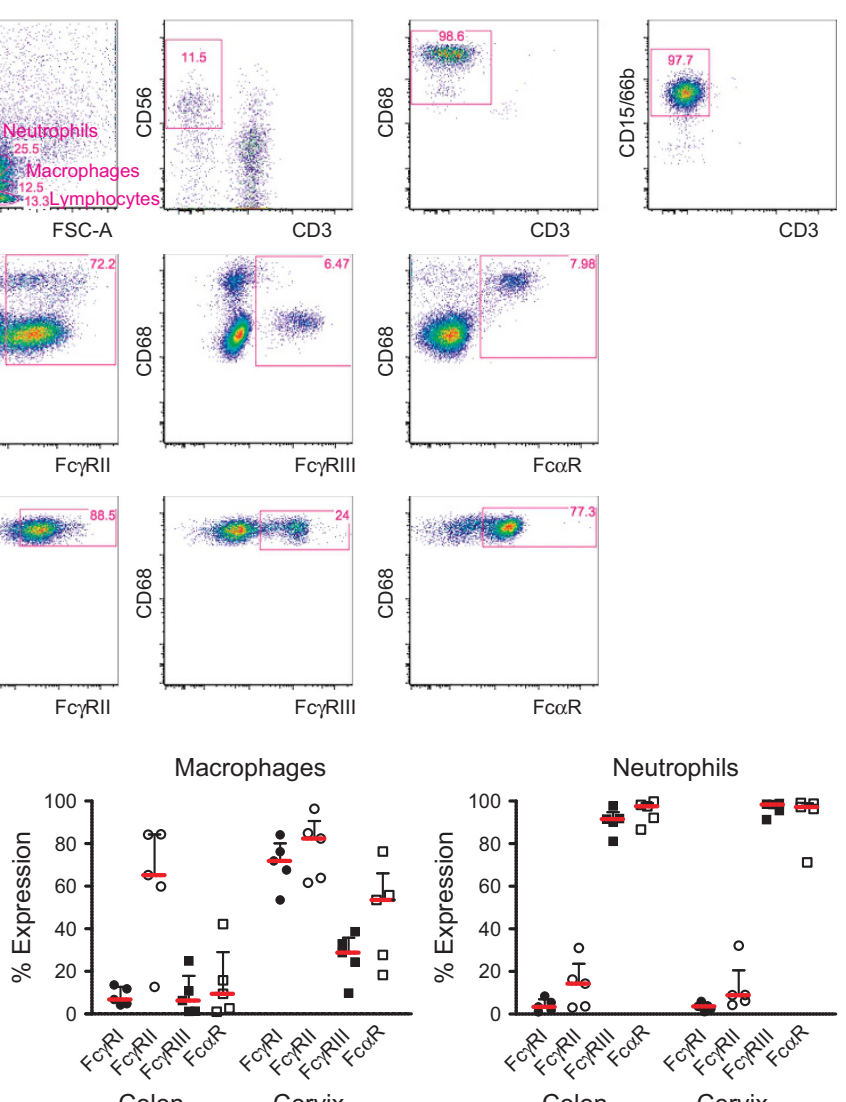

Colon

Colon
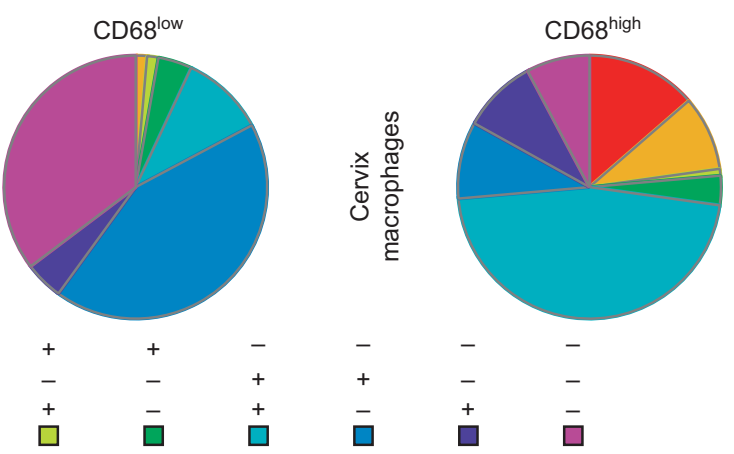

Figure 2 FcR expression profile on colonic and cervical innate immune cells. Gating strategy for natural killer (NK) cells, macrophages, and neutrophils is depicted for (a) colon and (b) cervix and a representative flow plot for each FcR is presented on (c) colonic and (d) cervical macrophages. Percent of FcR expression on NK cells, macrophages, and neutrophils (e) in colon and (f) cervix. The pie charts depict the coexpression profile of all Fc $\gamma$ receptors in colonic CD68 ${ }^{\text {high }}$ and $\mathrm{CD} 68^{\text {low }}$ and cervical CD68 ${ }^{\text {high }}$ macrophages.

Neutrophils were defined as small, highly granular, FSC ${ }^{\text {low }}$, $\mathrm{SSC}^{\text {high }}, \mathrm{CD} 45+, \mathrm{CD} 3-$, and $\mathrm{CD} 15+$ cells (Figure 2a,b). Both colonic and cervical neutrophils expressed FcR $\gamma$ III and $\mathrm{FcR} \alpha$ but negligible levels of $\mathrm{FcR} \gamma \mathrm{I}$ and FcR $\gamma \mathrm{II}$. Interestingly, whereas previous studies suggest that all neutrophils constitutively express Fc $\gamma$ RIIa, although at four- to fivefold lower levels compared with Fc $\gamma$ RIIIb, ${ }^{23}$ we only observed Fc $\gamma$ RII expression on $\sim 14 \%$ of colon-resident neutrophils and $\sim 9 \%$ of cervical neutrophils. Thus, here we report a dominant Fc $\gamma$ RIII and Fc $\alpha$ R profile on tissue-resident neutrophils. 


\section{Distinct mucosal phagocytic activity profiles}

As tissue-resident NK cells very rarely expressed Fc receptors (Figure 2e), suggesting poor antibody-directed functionality, we aimed to define the functional capacity of mucosal macrophages and neutrophils that frequently expressed Fc receptors. As both innate effector cell types are critically involved in the rapid phagocytic clearance of immune complexes in the blood, we evaluated the phagocytic potential of these cells from the different mucosal sites using a tissue phagocytosis assay. Gp120-coated fluorescent beads, complexed with a pool of $\mathrm{HIV}$-specific polyclonal antibodies (HIVIg, human immunodeficiency virus immunoglobulin), no antibodies, or a pool of nonspecific polyclonal antibodies from healthy individuals (IVIg, intravenous immunoglobulin), were added to mucosal cell suspensions (colon or cervical cells). Both neutrophil and macrophage phagocytic activity demonstrated a dose-dependent increase in phagocytic uptake in the presence of increasing HIV-specific antibodies and unaltered phagocytosis from the baseline level in the presence of nonspecific antibodies (Figure 3a-d). Interestingly, despite their lower frequencies, colonic neutrophils showed more robust phagocytic activity, significantly outperforming colonic macrophages (Figure 3a,c). In contrast, cervical macrophages exhibited identical phagocytic potentials as cervical neutrophils (Figure 3b,d), highlighting striking differences in macrophage, but not neutrophil activity, isolated from different mucosal sites. Given that neutrophils represent a much smaller fraction of total innate effector cells within the gut in healthy individuals (Figure 1), the contribution of both neutrophils and macrophages to absolute phagocytosis would likely be similar at the moment of transmission, where poor macrophage responsiveness will be counterbalanced by their sheer abundance.

\section{Unaltered cervical phagocytic activity in the presence of inflammatory cytokines associated with increased risk of HIV infection}

Epidemiological studies in young women in Africa suggest that sexually transmitted infections that cause inflammation are strongly associated with increased susceptibility to HIV infection. ${ }^{24}$ Specifically, elevated levels of macrophage inflammatory protein (MIP)- $1 \alpha$, MIP- $1 \beta$, interleukin (IL)-8, and interferon- $\gamma$-inducible protein-10 (IP-10) in the cervicovaginal lavage of women before infection have all been linked to increased risk of HIV infection. ${ }^{19}$ Moreover, MIP- $1 \alpha$, MIP- $1 \beta$, and IL- 8 are essential for the establishment of productive simian immunodeficiency virus infection in rhesus macaques. $^{25}$ Thus, we next sought to examine whether alterations in the inflammatory milieu might alter the phagocytic activity of mucosal-resident macrophages and neutrophils or whether vaccine or monoclonal therapeutic antibodies would continue to provide protection irrespective of inflammation. Cell suspensions from cervical mucosa were treated overnight with MIP-1 $\alpha$, MIP-1 $\beta$, IL-8, and IP-10, after which their a

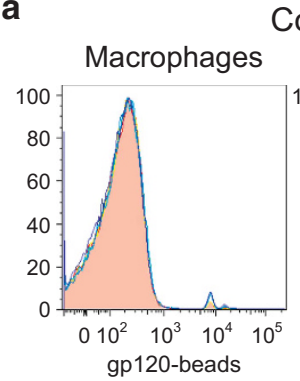

Colon

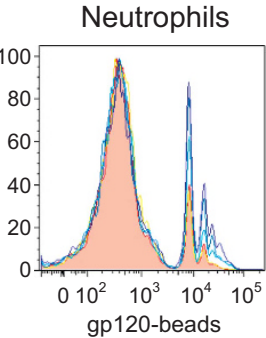

c

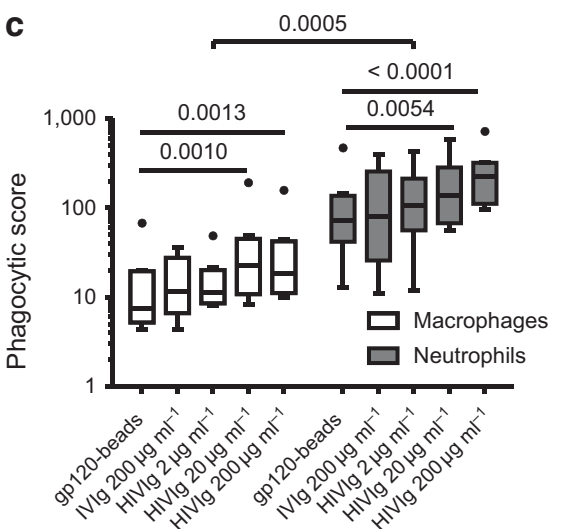

b

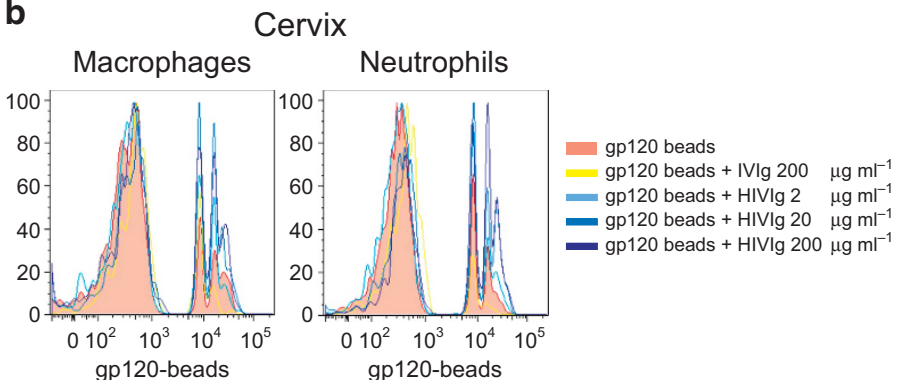

d

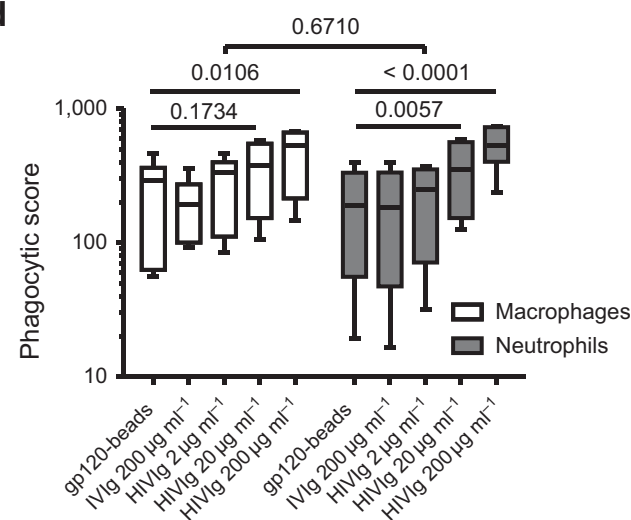

Figure 3 Phagocytic activity profiles in colon and cervix. Representative histograms of phagocytic activity of macrophages and neutrophils to gp120coated beads and gp120-directed immune complexes in (a) colon and (b) cervix. Phagocytic activity is represented as a phagocytic score $=\%$ of cells taking up beads $\times$ fluorescent intensity (MFI)/1,000 in (c) colon and (d) cervix. Colon, $N=8$; cervix, $N=6$. HIVlg, human immunodeficiency virus immunoglobulin; IVIg, intravenous immunoglobulin. 
a

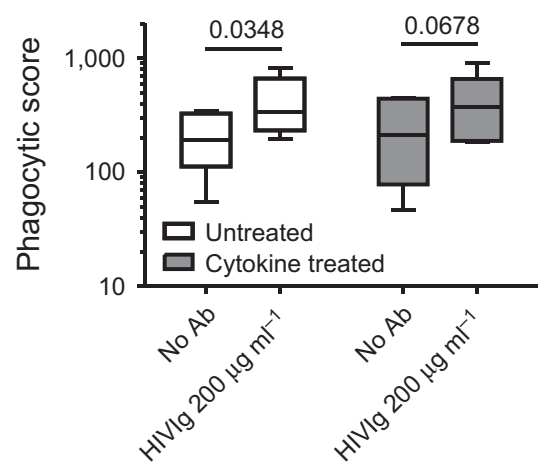

b

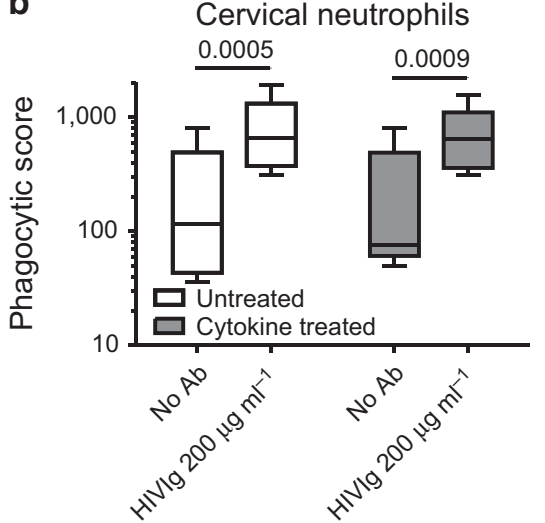

Figure 4 Phagocytic activity in cervix in the setting of inflammation. The phagocytic activity of (a) cervical macrophages and (b) cervical neutrophils treated with inflammatory cytokines: macrophage inflammatory protein (MIP)- $1 \alpha$, MIP-1 $\beta$, interleukin-8 (IL-8), and interferon- $\gamma$-inducible protein-10 (IP-10) as compared with untreated cells. Responses to gp120-beads and immune complexes (gp120-beads $+\mathrm{HIVlg}^{\text {at } 200 \mu \mathrm{g} \mathrm{ml}}{ }^{-1}$ ) are depicted. $\mathrm{Ab}$, antibody. $N=5$. HIVIg, human immunodeficiency virus immunoglobulin; IVIg, intravenous immunoglobulin.

phagocytic activity was determined. No differences in phagocytosis were observed between untreated and cytokine-treated cervical macrophages (Figure 4a) or cervical neutrophils (Figure 4b). These data suggest that cytokine exposure associated with an increased risk of HIV acquisition does not impair the phagocytic potential of tissue-resident phagocytes, rendering phagocytic activity an attractive mechanism to drive preventative immunity or therapeutic clearance in the setting of existing inflammation.

\section{Alterations in Fc effector cells in HIV infection}

In addition to their role in protection from infection, ${ }^{5,6}$ growing interest lies in the potential application of monoclonal therapeutics in HIV eradication. Importantly, the viral reservoir likely persists within the gut ${ }^{18}$ and the lymph nodes, specifically the germinal centers. ${ }^{17}$ Therefore, optimized monoclonal therapeutic strategies that are able to recruit local innate effector killing may drive more effective reservoir elimination. Given that previous studies have shown altered distribution of mucosal NK cell subsets in the gut in HIV infection, ${ }^{26}$ it is possible that other innate immune cells and $\mathrm{Fc}$ receptors may change during infection. Thus, we quantified the frequency of Fc $\gamma \mathrm{RII}+$ macrophages (Figure 5a,b) and Fc $\gamma$ RIII + neutrophils (Figure $\mathbf{5 c}, \mathbf{d}$ ) in gastrointestinal and lymph node sections from $27 \mathrm{HIV}$-infected subjects at different stages of disease and 15 seronegative controls. In lymph node and ileum, stable levels of macrophages were observed during HIV infection (Figure 5e,f). Similarly, neutrophil numbers were also constant in these compartments (Figure 5g,h); however, infiltrating neutrophils were observed in the lymph nodes in the early phase of HIV infection (documented seroconversion within the last 6 months), and in several subjects in the chronic stage of the disease (Figure 5h). Notably, these infiltrating neutrophils were observed in both the T-cell zone and B-cell follicles (Figure 5i,j,j), suggesting that these innate effector cells may gain access to the immunologic sanctuaries where the reservoir is thought to persist. Interest- ingly, this neutrophil expansion was strongly correlated with viremia (data not shown) and negatively correlated with absolute CD4 counts in acute, early, and chronic patients (Figure 5k), arguing that active viral replication, due to incomplete reservoir control, may drive the recruitment of neutrophils to the lymph node. In contrast to the associations observed for neutrophils, no link was observed between macrophage numbers and viremia in lymph nodes (data not shown). However, macrophage numbers were inversely associated with viremia in the rectum (Figure 5l) and ileum as compared with uninfected controls (data not shown).

Thus, given the selective recruitment of neutrophils to the germinal centers and stable, although at low levels in intestine, the application of Fc-enhancing monoclonal therapeutics that could selectively direct clearance of infected/reactivated cells may represent a promising novel means by which to eradicate the viral reservoir to attain functional cure. Furthermore, this study demonstrates that $\mathrm{Fc}$ receptor-expressing macrophages decline in the gastrointestinal mucosa but not in lymph nodes, suggesting their compromised effector cell potential for reservoir eradication at specific sites.

\section{Enhancing Fc effector activity to drive more effective antiviral activity}

The use of some of the most potent neutralizing antibodies to target and eliminate the viral reservoir is gaining momentum. ${ }^{27}$ However, although these antibodies clearly diminish viral loads transiently, ${ }^{11}$ they have demonstrated limited success in eliminating latently infected cells. Conversely, efforts in the monoclonal therapeutics community have shown that Fc effector function can be improved via Fc modifications. Many Fc modifications have now been identified, and each of these modifications can drive differential $F_{c}$ effector activities. ${ }^{28,29}$ Thus, we aimed to exploit these $\mathrm{Fc}_{\mathrm{c}}$ modifications to identify Fc enhancements that could augment the uptake of immune complexes by one of the most potent bnAbs, VRC01. ${ }^{30}$ The tissue phagocytosis assay was employed using immune 

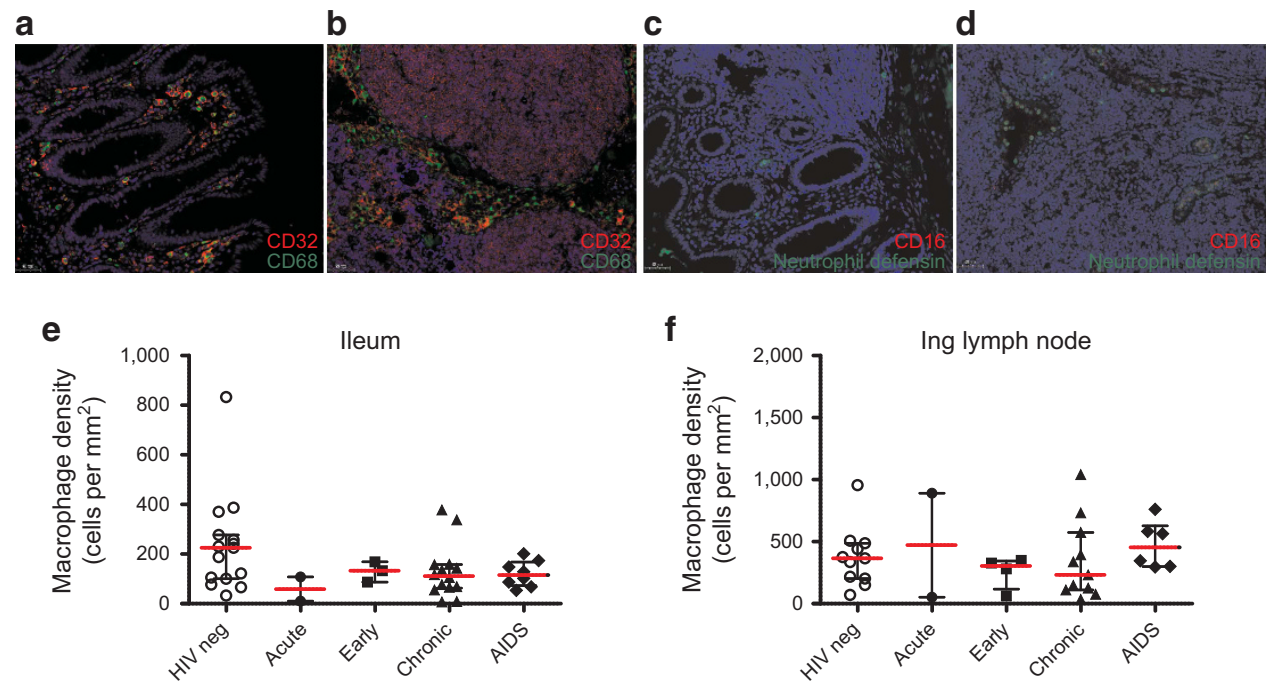

g

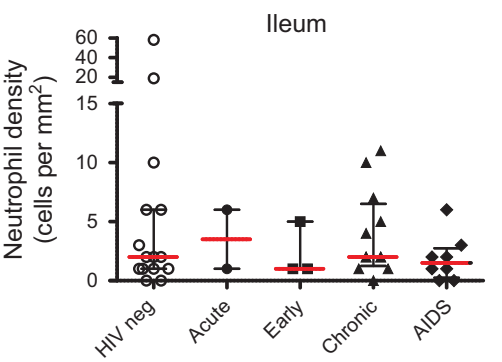

h
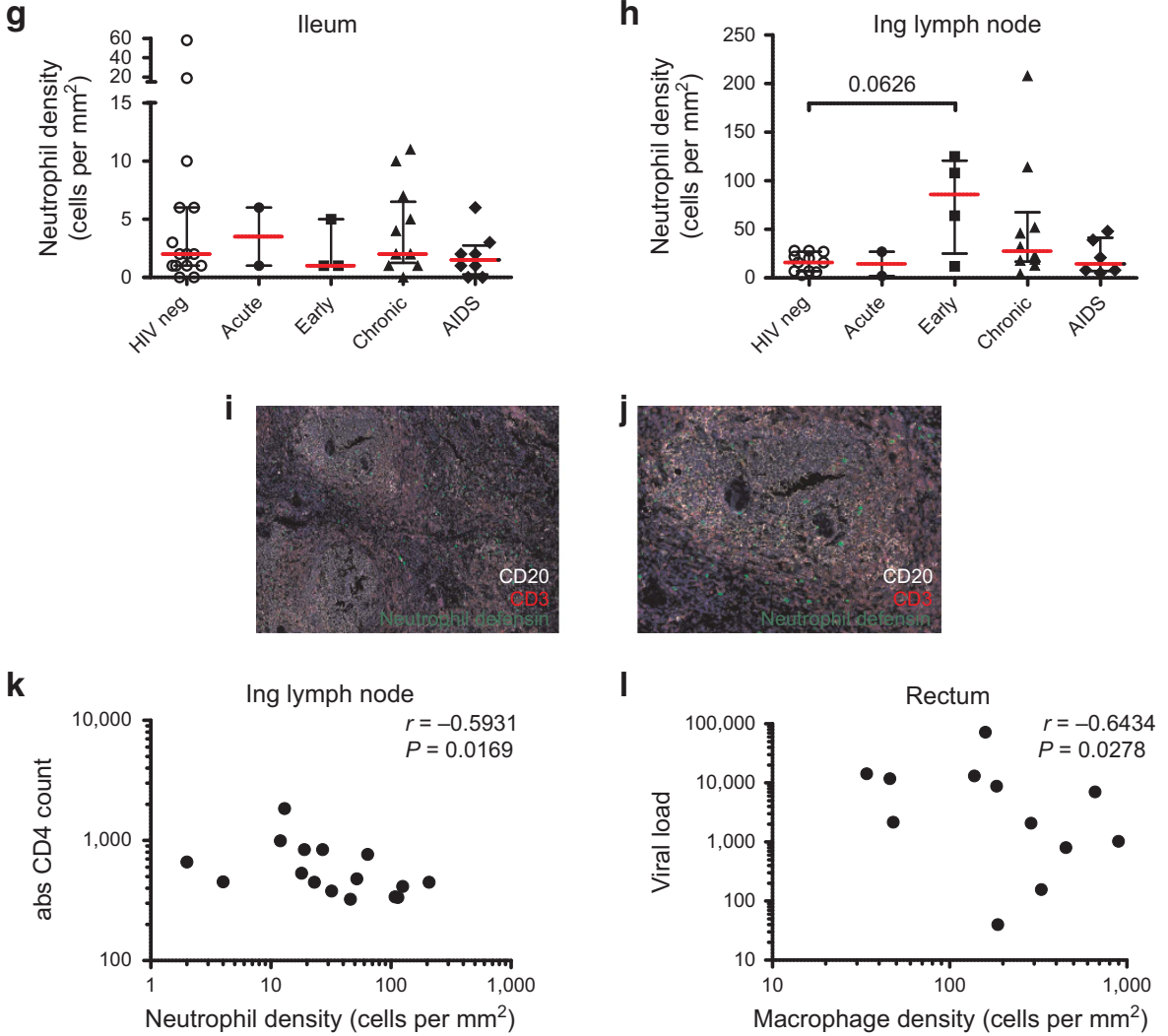

Figure 5 Changes in Fc effector-expressing innate immune cells in tissues during HIV infection. Immunofluorescence staining of CD32 + macrophages in (a) colon and (b) lymph node and CD16 + neutrophils in (c) colon and (d) lymph node. Quantification of (e, f) macrophages and (g, h) neutrophils in ileum and inguinal (Ing) lymph node in subjects at different stages of HIV disease (HIV neg, open circle; acute, closed circle; early, closed square; chronic, closed triangle; AIDS, closed diamond). (i, j) Infiltrating neutrophils into T-cell zone and B-cell follicles in HIV-infected lymph nodes. (k) Neutrophil density in lymph nodes correlated with absolute CD4 counts in acute, early, and chronically infected subjects. abs, absolute. (I) Macrophage density in rectal biopsies correlated negatively with peripheral viral load.

complexes generated with wild-type VRC01 or the following mutants: the S239D/I332E/G236A (SDIEGA) variant with enhanced FcR $\gamma$ IIA affinity, ${ }^{31}$ the S267E/H268F/S324T (SEHFST) variant with enhanced $\mathrm{Clq}$ affinity, ${ }^{32}$ and the S239D/I332E (SDIE) and S239D/I332E/A330L (SDIEAL) variants with enhanced FcR $\gamma$ IIIA affinity. ${ }^{31}$ All antibodies successfully boosted macrophage phagocytosis, and nearly all (with an exception of SEHFST variant) boosted neutrophil phagocytosis; however, the FcR $\gamma$ IIa-enhanced monoclonal variant, SDIEGA, was the most potent in enhancing phagocytosis of both innate immune cells (Figure 6a,b). Interestingly, the complement-enhancing variant, SEHFST, improved 

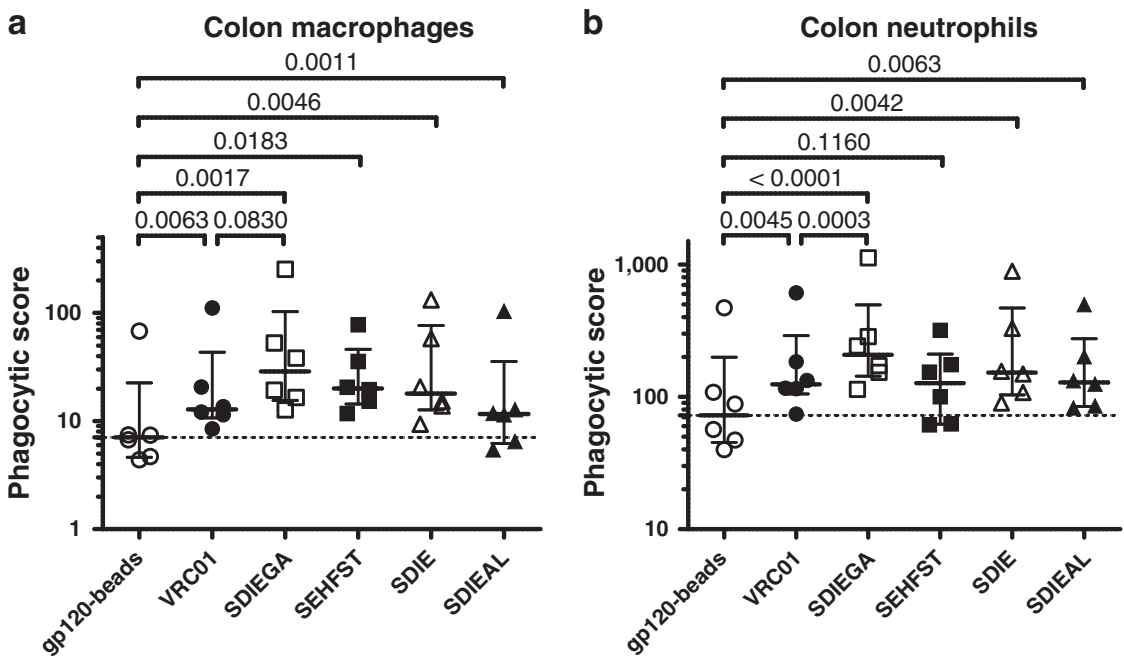

Figure 6 Enhancement of phagocytic activity via Fc engineering. Phagocytic activity of (a) macrophages and (b) neutrophils to immune complexes was evaluated using a VRC01 wild-type (WT) and its Fc-enhanced variants at $4 \mu \mathrm{g} \mathrm{ml}^{-1}$ (open circle, no antibody; closed circle, wild type VRC01; open square, SDIEGA, enhanced FcR $\gamma$ lla affinity; closed square, SEHFST, enhanced C1q (complement) affinity; open triangle, SDIE, enhanced FcR $\gamma$ Illa affinity; closed triangle, SDIEAL, enhanced FcR $\gamma$ Illa affinity). Phagocytic score is depicted.

macrophage, but not neutrophil phagocytosis, possibly because of expression of membrane $\mathrm{Clq}$ in gut macrophages ${ }^{33}$ or an increased affinity for Fc $\gamma$ RIIa and Fc $\gamma$ RIIb $^{34}$ of the S267E mutation. Nevertheless, none of the Fc enhancement strategies could improve colonic macrophage responsiveness to the levels observed in neutrophils, confirming the attenuated nature of macrophage potency in the gut environment and suggesting that harnessing the alternate innate immune effector cells may be critical for effective killing of virally infected cells in the gut.

Overall, the data presented here suggest that clearance of reactivated latent cells may be improved following the delivery of Fc-enhanced monoclonal therapeutics, specifically targeting phagocytes as effector cells in anti-HIV-1 prevention or cure strategies.

\section{DISCUSSION}

Although mounting evidence points to a critical role for antibody Fc effector function in protection from infection ${ }^{35}$ and eradication, ${ }^{10,11}$ knowledge about the principal tissue $\mathrm{FcR}+$ effector cells, their $\mathrm{Fc}$ receptor profile, functional activity, and their changes in infection is critical to maximize the effectiveness of antibody-mediated functions, capable of containing the infection at the site of HIV transmission and persistence. Here we found that although $\mathrm{FcR}+\mathrm{NK}$ cells were present at low frequencies in tissues, major differences in the distribution of $\mathrm{FcR}+$ macrophages and neutrophils were noticeable among the compartments. Specifically, we observed a dominant population of macrophages in the intestinal tract, lymph node, and endocervix in addition to large proportions of neutrophils in female genital tract, especially in the vaginal mucosa. Conversely, HIV infection was associated with reduced macrophage frequencies in intestinal tissues and increased neutrophil numbers in lymph nodes in the setting of detectable viremia. In addition, although neutrophil phagocytic activity was similar between the FRT and gastrointestinal tract, unaltered in the presence of inflammatory cytokines, colonic macrophages exhibited an attenuated functional profile compared with FRT macrophages. Promisingly, Fc-engineered monoclonal variants were able to improve both macrophage and neutrophil phagocytosis, providing a means to selectively promote these innate immune effector responses both in the setting of preventative and curative strategies. Overall, the study provides the first insights related to the specific innate immune Fc effector mechanisms in tissues that may confer the greatest level of protective or therapeutic immunity for future rationally designed interventions.

Accumulating data point to a role for antibody-dependent cell-mediated cytotoxicity (ADCC) in postinfection control of viremia in both humans ${ }^{36}$ and NHP. ${ }^{37,38}$ In contrast, the role of Fc-mediated functions in blocking HIV acquisition is indirect and more controversial, ${ }^{2,4,39}$ with the most provocative evidence emerging from the passive immunization studies employing neutralizing monoclonal antibodies, ${ }^{4,40}$ where the abrogation of $\mathrm{FcR}$ binding resulted in compromised protection from infection. ${ }^{4,41}$ More recently, Fc $\gamma$ R-mediated effector function was shown to contribute substantially to the in vivo capacity of several bnAbs to block viral entry, suppress viremia, and confer therapeutic activity. ${ }^{9}$ However, directed Fc receptor-enhancing strategies aimed at increasing protection from infection via ADCC, including the generation of nonfucosylated b12, resulting in improved affinity for FcR $\gamma$ IIIa and more effective in vitro NK cell-mediated killing, did not increase protection from HIV acquisition in an NHP low-dose challenge model. ${ }^{39}$ These results suggest that enhanced NK cell ADCC may not improve protective immunity, but instead other innate $\mathrm{Fc}$ effector mechanisms may be more critical. Along these lines, here we demonstrate that Fc $\gamma$ RIII + NK cells are nearly absent from most vulnerable tissues (Figure 2), supporting the notion that ADCC activity will depend heavily on the recruitment of peripheral blood NK cells to the site 
of viral invasion. Conversely, the employment of highly phagocytic cells that represent the first line of innate $\mathrm{FcR}$ activity may provide a rational means by which the first infectious virions and/or infected cells may be readily cleared before the establishment of a latent reservoir.

To date, little is known about the potential role of phagocytosis in blocking HIV acquisition. However, in vitro studies have demonstrated that HIV-specific antibodies are able to inhibit HIV in the presence of monocyte-derived macrophages. ${ }^{42}$ Moreover, Fc-mediated phagocytosis, but not ADCC, was directly associated with protection from a SHIV162P3 challenge following Ad26 vaccination of NHP. ${ }^{3}$ In addition, phagocytosis was also indirectly implicated in postinfection viral control by nonneutralizing antibodies in NHPs challenged vaginally with SHIV $162 \mathrm{p} 3{ }^{2}{ }^{2}$ Here we report highly enriched resident phagocytes at the sites of vulnerability (Figure 1). Although neutrophils were relatively infrequent within the gut lamina propria, these cells exhibited remarkably high phagocytosis (Figure 3), were often seen in vaginal epithelium, and were highly abundant in the venules perfusing all the tissues (Figure 1), potentially allowing for the rapid migration into the tissues upon infection.

Intriguingly, despite their high abundance in the gut, intestinal macrophages exhibited a log lower phagocytic activity than cervical macrophages, likely explained by distinct FcR expression profiles, where all FcRs were expressed on cervical macrophages, and gut macrophages predominantly expressed Fc $\gamma$ RII (Figure 2). These substantially different profiles reflect well-established phenotypic and functional heterogeneity of tissue-resident macrophages, reflective of their anatomic origin $^{43}$ and essential for their niche-specific functions. For example, intestinal macrophages play a critical role in the maintenance of gut homeostasis and the regulation of immune response to commensals. ${ }^{44}$ Thus, under steady-state conditions, lamina propria macrophages display an anergic phenotype, demonstrated by their inability to produce proinflammatory cytokines ${ }^{45}$ that may account for their poor responsiveness to phagocytosis. In contrast, FRT macrophages may be poised to respond to pathogens because of chronic exposure to non-self antigens, bacteria, and viruses, strongly pointing to their critical role as an innate effector that may confer enhanced protection in heterosexual exposure. Furthermore, these inherent differences in phagocytic activity observed in two distinct mucosal compartments could be directly linked to permeability of mucosal barriers to infection, where rectal mucosa is more permissive to viral transmission and systemic infection than vaginal mucosa as described in atraumatic SHIV infection model. ${ }^{46}$

Despite their low tissue-resident frequencies, neutrophils mediated the highest phagocytic activity (Figure 3), implicating these cells as potentially critical Fc effectors for both prevention and therapeutic strategies. Neutrophils are able to phagocytose bacteria, fungi, and virions. Yet, most of the work performed on neutrophils in HIV infection has focused on their role in viral pathogenesis. Circulating neutrophils constitutively express Fc $\gamma$ RIIa and four- to fivefold higher levels of Fc $\gamma$ RIIIb. ${ }^{23}$
Fc $\gamma$ RIIIb, the only glycosylphosphatidylinositol-anchored Fc receptor, is $97 \%$ homologous in its extracellular domain to Fc $\gamma$ RIIIa; however, Fc $\gamma$ RIIIb differs from Fc $\gamma$ RIIIa in its ability to drive phagocytosis. ${ }^{47}$ Although some studies suggest that Fc $\gamma$ RIIa drives the bulk of neutrophil phagocytic activity, ${ }^{48}$ the functional interaction between Fc $\gamma$ RIIIb and Fc $\gamma$ RIIa on neutrophils is unclear but likely collaborate to tune effector activity. ${ }^{49}$ Furthermore, neutrophils have been linked to vaccine-induced antiviral control of human papilloma virus in the FRT. ${ }^{50}$ Given that neutrophils are stable during the menstrual cycle and retain unaltered activity in inflammatory conditions (Figure 4), they may prove especially important in preventing heterosexual HIV transmission in women. Yet, because neutrophil activity was also potent in gut tissue (Figure 3), it is plausible that this innate immune effector cell may also contribute to the prevention of rectal HIV transmission.

To date, only one case of sterilizing cure has been reported; however, an array of different strategies are being explored to rapidly eliminate reactivated cells. ${ }^{51}$ Among them, efforts to utilize monoclonals have been tested. ${ }^{27}$ Specifically, combinations of 2G12, 2F5, and 4E10 monoclonal antibodies transiently controlled viremia in humans before viral escape, ${ }^{52}$ similar to the effect observed with $3 \mathrm{BNC117}$ in NHPs ${ }^{53}$ and humans. ${ }^{11}$ Nevertheless, studies in HIV-infected humanized mice demonstrate the clear need for $\mathrm{Fc}$ effector functions in reservoir control. ${ }^{54}$ Furthermore, recent data show that tissue myeloid cells, particularly within mesenteric lymph nodes and spleen, play a critical role in the phagocytosis of infected $\mathrm{T}$ cells; ${ }^{55}$ thus, particular Fc modifications that can enhance myeloid clearance mechanisms at the sites of virus persistence, including germinal centers, ${ }^{17}$ may enhance the elimination of the viral reservoir size. Moreover, $\mathrm{FcR}+$ neutrophils were present within the germinal centers (Figure 5) where they have been shown to collaborate in the induction of potent humoral immune responses. ${ }^{56}$ Along these lines, the Fc-mutant SDIEGA, known to exhibit enhanced affinity for Fc $\gamma$ RII, drove enhanced macrophage- and neutrophil-mediated phagocytosis compared with the wild-type bNAb (Figure 6), pointing to specific Fc modifications that could enhance reservoir eradication.

In conclusion, our data demonstrate that unlike NK cells, phagocytic cells represent our major mucosal tissue Fc receptor bearing effectors prepared to respond to antibodies for the rapid control and clearance of HIV. In addition, mucosal macrophages and neutrophils express Fc $\alpha$ R, pointing to further opportunities to harness both IgG and IgA molecules to harness the full innate armamentarium within vulnerable mucosal tissues.

\section{METHODS}

Subjects. Formalin-fixed, paraffin-embedded tissue slides from normal human colon, rectum, mesenteric lymph node, vagina, cervix, and uterus were obtained from the Massachusetts General Hospital Tissue Repository (Boston, MA). Fresh HIV-negative tissue samples from colon and cervix, collected from healthy regions, outside of 
cancerous lesions, were obtained through the Ragon Institute Tissue Platform (Cambridge, MA). Biopsy samples from rectum, ileum, and inguinal lymph nodes from HIV + donors and HIV - controls were obtained from the University of Minnesota (Minneapolis, MN). Tissue samples were unmatched (i.e., rectum, ileum, and lymph node were collected from independent donors) and therefore each tissue type was treated independently in the analysis. All HIV + patients were treated with antiretroviral therapy and were biopsied at different stages of infection (acute, early, chronic, or AIDS). In all, 13 rectal biopsies (all from HIV + subjects), 42 ileal biopsies (15 uninfected HIV - controls and 27 HIV + subjects), and 35 inguinal lymph node biopsies (11 uninfected HIV - controls and 24 HIV + subjects) were analyzed. Viral load and CD4 counts were measured at the time of biopsy. All tissue donors were adults between 20 and 63 years of age $($ median $=40)$. All samples were analyzed in a blinded manner.

Immunohistochemistry. Antigen retrieval and signal detection were performed as previously described. ${ }^{26}$ The following primary Abs were used: goat polyclonal anti-NKp46 (R\&D Systems, Minneapolis, MN), mouse anti-CD56 (clone 123C3, Dako, Carpinteria, CA), mouse antiCD68 (clone KP1, Dako), mouse anti-neutrophil defensins (clone D21, Leica Biosystems, Buffalo Grove, IL), mouse anti-CD16 (clone 2H7, Leica Biosystems), rabbit anti-CD32 (clone EPR6657(2), Abcam, Cambridge, MA), mouse anti-CD20 (clone L26, Dako), and rabbit polyclonal anti-CD3 (Dako). The following control Abs were used: mouse IgG1 (Dako), goat IgG (R\&D Systems), mouse IgG2a (Abcam), and rabbit IgG (Abcam).

Immunofluorescence. Antigen-retrieval and autofluorescence masking were performed as previously described. ${ }^{26}$ The primary and control Abs were used as above. The following detection Abs were used: goat anti-mouse IgG1 Alexa Fluor 488, goat anti-rabbit IgG Alexa Fluor 594, and goat anti-mouse IgG2a Alexa Fluor 647 (Life Technologies, Grand Island, NY).

Microscopy. Slides were scanned using a Zeiss MIRAX MIDI slide scanner in the brightfield mode or a TissueFAXS (Tissue Gnostics, based on a Zeiss Axio Imager Z2 microscope, Jena, Germany) in the fluorescence mode.

Image analysis. Cell counts were performed using the TissueQuest analysis software (Tissue Gnostics, Vienna, Austria) on fluorescence images.

Flow cytometry. Flow cytometric evaluation of Fc receptor expression was performed on freshly isolated cells from enzymatically digested colon and cervical tissues. $1 \times 10^{6}$ colon or $1 \times 10^{5}$ cervical cells were stained in two panels with anti-CD3 Alexa Fluor 700 (clone UCHT1), anti-CD56 PE-Cy7 (clone NCAM16.2), anti-CD64 FITC (clone 10.1), anti-CD89 PE (clone A59), anti-CD45 PE-Cy5 (clone HI30), antiCD15 PacificBlue (clone W6D3), anti-CD16 BV510 (clone 3G8), antiCD32 APC (clone FUN-2), anti-CD64 R-PE (clone 10.1, Dako), and blue viability dye (Life Technologies). Cells were washed and fixed with Perm A buffer (Life Technologies). Intracellular staining with antiCD68 FITC (clone KP1, Dako) was performed in the presence of permeabilization buffer (Perm B, Life Technologies). The data were acquired using an LSRII flow cytometer (BD Biosciences, Franklin Lakes, NJ) and analyzed using FlowJo (Ashland, OR) and SPICE version 5.1 (NIAID, Rockville, MD).

Generation of VRC01 variants. Antibody variants were expressed as previously described. ${ }^{57}$ In brief, $\mathrm{CMV} / \mathrm{R}$ mammalian expression vectors for the VRC- 01 IgG1 light and heavy chains were obtained from the NIH AIDS Reagent Program (Germantown, MD). Following sequence verification, DNA was isolated via maxi-prep (Qiagen, Germantown, MD) and used to transfect suspension cultures of human embryonic kidney (HEK) 293F cells grown in Freestyle media (Invitrogen, Grand Island, NY) using $25 \mathrm{kD}$ branched polyethylenimine (PolySciences, Warrington, PA). Antibodies were transiently expressed for 7 days at $37^{\circ} \mathrm{C}, 5 \% \mathrm{CO}_{2}$. Fc domain amino acid point mutations were incorporated via QuikChange PCR (Stratagene, La Jolla, CA). Sequences for each construct have been previously described. ${ }^{57}$

Tissue phagocytosis assay. A tissue phagocytosis assay was developed based on a previously described THP1-based phagocytosis assay. ${ }^{58}$ In brief, biotinylated gp120 antigen was incubated with $1 \mu \mathrm{m}$ diameter red fluorescent neuravidin beads (Life Technologies) at a 4:1 protein/bead ratio ( $4 \mu \mathrm{g}$ protein/1 $\mu \mathrm{l}$ bead suspension) overnight at $4{ }^{\circ} \mathrm{C}$. Beads were subsequently washed twice in phosphate-buffered saline/ $1 \%$ bovine serum albumin in order to remove excess unbound antigen, and then resuspended at a final dilution of 1:100 in phosphatebuffered saline/bovine serum albumin. Immune complexes were formed by incubating gp120-coated beads with antibodies for $2 \mathrm{~h}$ at $37^{\circ} \mathrm{C}$ : different doses of HIV-IG (NIH AIDS Reagent Program, Division of AIDS, NIAID, NIH: Catalog 3957, HIV-IG from NABI and NHLBI), VRC01 wild-type and VRC01 Fc mutants: FcR $\gamma I I a-$ enhancing S239D/I332E/G236A (SDIEGA), ${ }^{31}$ complement-enhancing S267E/H268F/S324T (SEHFST), ${ }^{32}$ and two FcR $\gamma$ IIIa-enhancing S239D/I332E $(\mathrm{SDIE})^{31}$ and S239D/I332E/A330L (SDIEAL). ${ }^{39}$ Immune complexes were then cocultured with $1 \times 10^{6}$ colonic or $1 \times 10^{5}$ cervical cells for $2 \mathrm{~h}$ at $37^{\circ} \mathrm{C}$. In addition, inflammatory cytokines and chemokines (recombinant human MIP- $1 \alpha$, recombinant human MIP-1 $\beta$, recombinant human IL-8, and recombinant human IP-10, R\&D Systems), each at $200 \mathrm{ng} \mathrm{ml}^{-1}$, were added to cervical mucosa in a subset of experiments to induce an inflammatory environment before coculture with immune complexes. All cells were washed and stained with anti-CD45 BV510 (clone HI30), anti-CD3 Alexa Fluor 700 (clone UCHT1), anti-CD56-PE-Cy7 (clone NCAM16.2), anti-66b Pacific Blue (clone G10F5), blue viability dye, and anti-CD68 FITC (clone KP1). The data were acquired using a BD LSRII flow cytometer. Bead uptake was evaluated by FlowJo analysis and phagocytic score ( $\%$ cells taking up beads $\times$ mean fluorescent intensity/1,000) was calculated.

Statistics. Descriptive measures (median, interquartile range, frequency, and percent) were used to summarize the data. Logarithmic transformation was performed to facilitate normalization of the data distribution. Two-way analysis of variance with Dunn's post hoc analyses were used to compare phagocytosis between cell types stimulated by different conditions (gp120-beads alone or immune complexes) and to compare the effect of cytokine treatment on cells stimulated by different conditions. One-way analysis of variance with Dunn's post hoc analyses were used to compare groups of uninfected and HIV-infected individuals with controlled or uncontrolled viremia and subjects at different stages of HIV disease (acute, early, chronic, AIDS, and uninfected controls) to test differences in cellular counts. Spearman's rank correlations were used to examine bivariate associations between cellular counts and viral load or CD4 count. Paired $t$-tests were used to compare phagocytic activity induced by gp120beads and immune complexes formed by VRC01 antibody and different VRC01 Fc mutants. Reported $P$-values are two sided and values of $P \leq 0.05$ were considered significant. Statistical analysis and graphing were performed using GraphPad Prism Software (La Jolla, CA).

\section{ACKNOWLEDGMENTS}

We thank Joseph Misdraji, Joelle Brown and Eric Safai for their help with healthy control tissue collection. Furthermore, we thank summer students Fernanda Cerqueira and Madelyn O'Kelley-Bangsberg for help with optimization of immunohistochemistry stainings and Ciprian Husanu for help with the Tissue Gnostics software. This work was supported by the National Institute of Health (R01 Al080289, R21 Al110165, and Al102660), the Bill and Melinda Gates Foundation CAVD (OPP1032817: Leveraging Antibody Effector Function), and the Ragon Institute of MGH, MIT, and Harvard. 


\section{DISCLOSURE}

The authors declared no conflict of interest.

c) 2016 Society for Mucosal Immunology

\section{REFERENCES}

1. Haynes, B.F. et al. Immune-correlates analysis of an HIV-1 vaccine efficacy trial. N. Engl. J. Med. 366, 1275-1286 (2012).

2. Moog, C. et al. Protective effect of vaginal application of neutralizing and nonneutralizing inhibitory antibodies against vaginal SHIV challenge in macaques. Mucosal Immunol. 7, 46-56 (2014).

3. Barouch, D.H. et al. Protective efficacy of a global HIV-1 mosaic vaccine against heterologous SHIV challenges in rhesus monkeys. Cell 155, 531-539 (2013).

4. Hessell, A.J. et al. Fc receptor but not complement binding is important in antibody protection against HIV. Nature 449, 101-104 (2007).

5. Zeitlin, L. et al. Enhanced potency of a fucose-free monoclonal antibody being developed as an Ebola virus immunoprotectant. Proc. Natl. Acad. Sci. USA 108, 20690-20694 (2011).

6. DiLillo, D.J., Tan, G.S., Palese, P. \& Ravetch, J.V. Broadly neutralizing hemagglutinin stalk-specific antibodies require FcgammaR interactions for protection against influenza virus in vivo. Nat. Med. 20, 143-151 (2014).

7. Forthal, D.N. et al. Antibody-dependent cellular cytotoxicity independently predicts survival in severely immunocompromised human immunodeficiency virus-infected patients. J. Infect. Dis. 180, 1338-1341 (1999).

8. Sawyer, L.A. et al. Possible beneficial effects of neutralizing antibodies and antibody-dependent, cell-mediated cytotoxicity in human immunodeficiency virus infection. AIDS Res. Hum. Retroviruses 6, 341-356 (1990).

9. Halper-Stromberg, A. et al. Broadly neutralizing antibodies and viral inducers decrease rebound from HIV-1 latent reservoirs in humanized mice. Cell 158, 989-999 (2014).

10. Barouch, D.H. et al. Therapeutic efficacy of potent neutralizing HIV-1specific monoclonal antibodies in SHIV-infected rhesus monkeys. Nature 503, 224-228 (2013).

11. Caskey, M. et al. Viraemia suppressed in HIV-1-infected humans by broadly neutralizing antibody 3BNC117. Nature 522, 487-491 (2015).

12. Fridman, W.H. Fc receptors and immunoglobulin binding factors. FASEB J. 5, 2684-2690 (1991).

13. Burton, D.R. Antibodies, viruses and vaccines. Nat. Rev. Immunol. 2 , 706-713 (2002).

14. Carrega, P. \& Ferlazzo, G. Natural killer cell distribution and trafficking in human tissues. Front. Immunol. 3, 347 (2012).

15. Tuijnman, W.B., Van Wichen, D.F. \& Schuurman, H.J. Tissue distribution of human IgG Fc receptors CD16, CD32 and CD64: an immunohistochemical study. APMIS 101, 319-329 (1993).

16. Centers for Disease Control and Prevention. Monitoring selected national HIV prevention and care objectives by using HIV surveillance data-United States and 6 U.S. dependent areas-2012. HIV Surveillance Supplemental Report 201419 ((No. 3)2014).

17. Fukazawa, Y. et al. B cell follicle sanctuary permits persistent productive simian immunodeficiency virus infection in elite controllers. Nat. Med. 21, 132-139 (2015).

18. Chun, T.W. et al. Persistence of HIV in gut-associated lymphoid tissue despite long-term antiretroviral therapy. J. Infect. Dis. 197, 714-720 (2008).

19. Masson, L. et al. Genital inflammation and the risk of HIV acquisition in women. Clin. Infect. Dis. 61, 260-269 (2015).

20. Summers, C. et al. Neutrophil kinetics in health and disease. Trends Immunol. 31, 318-324 (2010).

21. Leno-Duran, E., Munoz-Fernandez, R., Olivares, E.G. \& Tirado-Gonzalez, I. Liaison between natural killer cells and dendritic cells in human gestation. Cell. Mol. Immunol. 11, 449-455 (2014).

22. Metes, D. et al. Expression of functional CD32 molecules on human NK cells is determined by an allelic polymorphism of the FcgammaRIIC gene. Blood 91, 2369-2380 (1998).

23. Selvaraj, P., Rosse, W.F., Silber, R. \& Springer, T.A. The major Fc receptor in blood has a phosphatidylinositol anchor and is deficient in paroxysmal nocturnal haemoglobinuria. Nature 333, 565-567 (1988).
24. Masson, L. et al. Defining genital tract cytokine signatures of sexually transmitted infections and bacterial vaginosis in women at high risk of HIV infection: a cross-sectional study. Sex. Transm. Infect. 90, 580-587 (2014).

25. Li, Q. et al. Glycerol monolaurate prevents mucosal SIV transmission. Nature 458, 1034-1038 (2009).

26. Sips, M. et al. Altered distribution of mucosal NK cells during HIV infection. Mucosal Immunol. 5, 30-40 (2012).

27. Euler, Z. \& Alter, G. Exploring the potential of monoclonal antibody therapeutics for HIV-1 eradication. AIDS Res. Hum. Retroviruses 31, 13-24 (2015).

28. Jefferis, R., Lund, J. \& Pound, J.D. IgG-Fc-mediated effector functions: molecular definition of interaction sites for effector ligands and the role of glycosylation. Immunol. Rev. 163, 59-76 (1998).

29. Lazar, G.A. et al. Engineered antibody Fc variants with enhanced effector function. Proc. Natl. Acad. Sci. USA 103, 4005-4010 (2006).

30. Li, Y. et al. Mechanism of neutralization by the broadly neutralizing HIV-1 monoclonal antibody VRC01. J. Virol. 85, 8954-8967 (2011).

31. Richards, J.O. et al. Optimization of antibody binding to FcgammaRlla enhances macrophage phagocytosis of tumor cells. Mol. Cancer Ther. 7 , 2517-2527 (2008)

32. Moore, G.L., Chen, H., Karki, S. \& Lazar, G.A. Engineered Fc variant antibodies with enhanced ability to recruit complement and mediate effector functions. MAbs 2, 181-189 (2010).

33. Kaul, M. \& Loos, M. Expression of membrane C1q in human monocytederived macrophages is developmentally regulated and enhanced by interferon-gamma. FEBS Lett. 500, 91-98 (2001).

34. Smith, P., DiLillo, D.J., Bournazos, S., Li, F. \& Ravetch, J.V. Mouse model recapitulating human Fcgamma receptor structural and functional diversity. Proc. Natl. Acad. Sci. USA 109, 6181-6186 (2012).

35. Chung, A.W. et al. Polyfunctional Fc-effector profiles mediated by IgG subclass selection distinguish RV144 and VAX003 vaccines. Sci. Transl. Med. 6, 228ra38 (2014).

36. Lewis, G.K. Role of Fc-mediated antibody function in protective immunity against HIV-1. Immunology 142, 46-57 (2014).

37. Ferrantelli, F. et al. Post-exposure prophylaxis with human monoclonal antibodies prevented SHIV89.6P infection or disease in neonatal macaques. AIDS 17, 301-309 (2003).

38. Banks, N.D., Kinsey, N., Clements, J. \& Hildreth, J.E. Sustained antibodydependent cell-mediated cytotoxicity (ADCC) in SIV-infected macaques correlates with delayed progression to AIDS. AIDS Res. Hum. Retroviruses 18, 1197-1205 (2002).

39. Moldt, B. et al. A nonfucosylated variant of the anti-HIV-1 monoclonal antibody b12 has enhanced FcgammaRIlla-mediated antiviral activity in vitro but does not improve protection against mucosal SHIV challenge in macaques. J. Virol. 86, 6189-6196 (2012).

40. Bournazos, S. et al. Broadly neutralizing anti-HIV-1 antibodies require Fc effector functions for in vivo activity. Cell 158, 1243-1253 (2014).

41. Hessell, A.J. et al. Effective, low-titer antibody protection against low-dose repeated mucosal SHIV challenge in macaques. Nat. Med. 15, 951-954 (2009).

42. Holl, V. et al. Involvement of Fc gamma RI (CD64) in the mechanism of HIV-1 inhibition by polyclonal IgG purified from infected patients in cultured monocyte-derived macrophages. J. Immunol. 173, 6274-6283 (2004).

43. Ortiz, A.M., DiNapoli, S.R. \& Brenchley, J.M. Macrophages are phenotypically and functionally diverse across tissues in simian immunodeficiency virus-infected and uninfected Asian macaques. J. Virol. 89, 5883-5894 (2015).

44. Zigmond, E. \& Jung, S. Intestinal macrophages: well educated exceptions from the rule. Trends Immunol. 34, 162-168 (2013).

45. Smythies, L.E. et al. Human intestinal macrophages display profound inflammatory anergy despite avid phagocytic and bacteriocidal activity. J. Clin. Invest. 115, 66-75 (2005).

46. Chenine, A.L. et al. Relative transmissibility of an R5 clade C simian-human immunodeficiency virus across different mucosae in macaques parallels the relative risks of sexual $\mathrm{HIV}-1$ transmission in humans via different routes. J. Infect. Dis. 201, 1155-1163 (2010).

47. Nagarajan, S. et al. Ligand binding and phagocytosis by CD16 (Fc gamma receptor III) isoforms. Phagocytic signaling by associated zeta and 
gamma subunits in Chinese hamster ovary cells. J. Biol. Chem. 270, 25762-25770 (1995).

48. Rivas-Fuentes, S., Garcia-Garcia, E., Nieto-Castaneda, G. \& Rosales, C. Fcgamma receptors exhibit different phagocytosis potential in human neutrophils. Cell. Immunol. 263, 114-121 (2010).

49. Chen, K. et al. Endocytosis of soluble immune complexes leads to their clearance by FcgammaRIIIB but induces neutrophil extracellular traps via FcgammaRIIA in vivo. Blood 120, 4421-4431 (2012).

50. Day, P.M. et al. In vivo mechanisms of vaccine-induced protection against HPV infection. Cell Host Microbe 8, 260-270 (2010).

51. Deeks, S.G. et al. Towards an HIV cure: a global scientific strategy. Nat. Rev. Immunol. 12, 607-614 (2012).

52. Trkola, A. et al. Delay of HIV-1 rebound after cessation of antiretroviral therapy through passive transfer of human neutralizing antibodies. Nat. Med. 11, 615-622 (2005).
53. Shingai, M. etal. Antibody-mediated immunotherapy of macaques chronically infected with SHIV suppresses viraemia. Nature 503, 277-280 (2013).

54. Klein, F. et al. HIV therapy by a combination of broadly neutralizing antibodies in humanized mice. Nature 492, 118-122 (2012).

55. Calantone, N. et al. Tissue myeloid cells in SIV-infected primates acquire viral DNA through phagocytosis of infected T cells. Immunity 41, 493-502 (2014).

56. Puga, I. et al. B cell-helper neutrophils stimulate the diversification and production of immunoglobulin in the marginal zone of the spleen. Nat. Immunol. 13, 170-180 (2012).

57. Boesch, A.W. et al. Highly parallel characterization of lgG Fc binding interactions. MAbs 6, 915-927 (2014).

58. Ackerman, M.E. et al. A robust, high-throughput assay to determine the phagocytic activity of clinical antibody samples. J. Immunol. Methods 366, 8-19 (2011). 